Trinity University

Digital Commons @ Trinity

Psychology Faculty Research

Psychology Department

$11-2016$

\title{
Judging Knowledge in the Digital Age: The Role of External- Memory Organization
}

Kristy A. Hamilton

Trinity University, khamilto@trinity.edu

Kevin Mclntyre

Trinity University, kmcintyr@trinity.edu

Paula T. Hertel

Trinity University, phertel@trinity.edu

Follow this and additional works at: https://digitalcommons.trinity.edu/psych_faculty

Part of the Psychology Commons

Publication Details

Applied Cognitive Psychology

\section{Repository Citation}

Hamilton, K. A., McIntyre, K. P., \& Hertel, P. T. (2016). Judging knowledge in the digital age: The role of external-memory organization. Applied Cognitive Psychology, 30(6), 1080-1087. doi: 10.1002/acp.3277

This Post-Print is brought to you for free and open access by the Psychology Department at Digital Commons @ Trinity. It has been accepted for inclusion in Psychology Faculty Research by an authorized administrator of Digital Commons@ Trinity. For more information, please contact jcostanz@trinity.edu. 
Judging Knowledge in the Digital Age: The Role of External-Memory Organization Kristy A. Hamilton, Kevin P. McIntyre, and Paula T. Hertel Trinity University

Word count for main body and references: 4938 (Short Report)

\section{Author Note}

Kristy A. Hamilton, Kevin P. McIntyre, and Paula T. Hertel, Department of Psychology, Trinity University

We thank Thomas Coverdale for assistance in the formulation and execution of these studies. Kristy Hamilton was funded by a Murchison summer fellowship at Trinity University. Correspondence concerning this article should be addressed to Paula Hertel, Department of Psychology, Trinity University, San Antonio, TX 78212.

Email: phertel@trinity.edu 


\begin{abstract}
Two studies examined relations between features of external-memory repositories (personal computers) and confidence in knowing. Participants judged their confidence in knowledge related to their work or studies and then answered questions about the way they store and use information. Participants who maintained more organized repositories were more confident in their knowledge. Furthermore, moderation analyses showed that the participants who navigated through their files by manually clicking through folders to find documents, but not those who use an automated search feature, felt more knowledge confident if they maintained a well-organized electronic repository. These results provide evidence for relation between assessments of internally "stored" knowledge and the degree of organization of their externally stored "knowledge."
\end{abstract}

Keywords: external memory, organization, feeling of knowing, metacognition 
Judging Knowledge in the Digital Age: The Role of External-Memory Organization

When technology transforms the scope of human faculty, the distinction between features belonging to oneself and the external environment begs reconsideration. In fact, throughout history, new technologies have extended the scope of basic human faculties in ways that are pervasive - the wheel, for example, extends the scope of our feet, and clothes extend the scope of our skin. The extension of mind by technology—-from stone tablets to computers - has occurred so gradually and pervasively that accurate distinctions between internally and externally held knowledge are difficult to make and sometimes seem irrelevant (Hertel, 1993; Ward, 2013). The present investigation examined the relation between confidence in knowing and features of external repositories, in this case personal computers and their memory extensions. Our primary hypothesis was that the degree to which individuals maintain organized external repositories can predict their confidence in knowledge related to their main intellectual enterprise. In essence, we propose that external memory organization operates as a heuristic, similarly to the availability heuristic (Tversky \& Kahneman, 1973). The availability heuristic in judgment and decision-making tasks is a short-cut method of relying on the ease with which a few initial instances come to mind (the ease of accessibility). The unarticulated assumption is something like: If I can easily think of examples of X, X must be true (or more prevalent). Judgments of personal knowledge are also based on retrieval fluency (see Kelley \& Lindsay, 1993). For example, Winkielman, Schwarz, and Belli (1998) asked participants to recall either 4 or 12 childhood memories (the easy and difficult conditions) before rating the completeness of their memories representing those childhood years. Despite recalling many fewer memories, the participants in the easy condition gave higher ratings. Retrieval difficulty in the 12-memories 
condition led participants more frequently to infer that large parts of their childhood memories were missing.

The logic of an organization heuristic is similar: When individuals judge personal knowledge, they implicitly assess the accessibility of that knowledge but without careful attention to whether related information is stored internally or externally (Mitchell \& Johnson, 2000). And because organization increases success of retrieval from organic memory (Bower, 1970), individuals rely on a general sense of the organization of all sources of information belonging to them, organic or inorganic, as an indicator that the information can be found and is therefore as good as known.

Past research has shown that information stored in well-organized external repositories characterize confidence in knowledge within specific domains. For example, Hertel (1988) asked faculty members to judge their confidence in knowledge related to their domain of academic research and to describe features of their non-digital external repositories (file-drawers and bookshelves). Faculty members who had stored more information and maintained betterorganized offices reported greater confidence in their research-related knowledge. Furthermore, multiple-regression analyses indicated that organization was the strongest predictor and accounted for confidence differences even after considering the relation with crude measures of amount of knowledge stored externally. Recent research has expanded this finding to include information stored on the internet by showing that access to the internet influences judgments of one's cognitive abilities. Ward (2013) found that individuals who were better able to use the internet to search for answers to trivia questions scored significantly higher on a survey of Cognitive Self-Esteem (CSE), but not general self-esteem measures, indicating that they were more confident in their ability to think about, remember, and locate information. Thus, relying on 
external storage devices, paper or electronic, can blur distinctions between internally and externally stored knowledge (a storage version of reality-monitoring errors, Johnson \& Raye, 1981).

Although some studies have shown that features of internet use are associated with judgments of cognitive abilities (e.g., Fisher, Goddu, \& Keil, 2015; Ward, 2013), little research has examined the relation between features of personal electronic repositories and judgments of knowledge. Files on a personal computer perhaps can be even more easily seen as extensions of organic memory than can places on the public internet, shared with others. External files placed within a private space (i.e., objects that are 'mine') are more likely to be incorporated into one's sense of self (Kim \& Johnson, 2013). Furthermore, since the integration of technology into everyday lives, we know of no examination of the relation between organizational features of external repositories and judgments of knowledge.

The primary goals of the current research were to replicate and extend the finding concerning a relation between confidence in knowledge and organizational features of external memory (Hertel, 1988). With the goal of replication in mind, we predicted that participants who report better-organized repositories would also report greater confidence in knowledge related to their work or studies. Because correlational evidence of this kind is consistent with the use of an organization heuristic but ultimately is limited in its ability to determine whether organization leads to confidence or whether knowledgeable people are simply more confident and more organized, we also predicted that external memory organization would be used only to the extent that the method used to retrieve electronic information actually requires an organized repository. Therefore, to extend Hertel's findings, the second goal specifically takes into account the nature of electronic search techniques. Whereas manually searching through nested folders requires 
those folders to be organized if one is to quickly and accurately find files, key-word searches do not. Thus, we predicted that the relation between perceived organization and knowledge confidence would be stronger for individuals who navigate through their files by manually clicking through folders than for those who use an automated search feature to find their files. If true expertise causes both greater confidence and better organization, it would be hard to understand how this causal relation would be restricted only to cases in which the experts use nested folders and search by accessing that structure.

In addition to the main predictions, we also addressed related issues. In Study 1 we asked questions related to files stored on the desktop. The amount of information stored electronically corresponds to an important set of predictors in the study by Hertel (1988); unlike files on a hard drive or cloud device, the number of files on a desktop could be easily counted in an on-line study. Moreover, we suspected that the way individuals store files on their desktop would at least partly reflect their organizational tendencies because the desktop is the primary display screen of a user interface. Therefore we asked participants to count their desktop files and broadly characterize desktop organization. In Study 2 we asked questions about keeping and deleting files, thinking that there were two different possible outcomes related to our concerns. In one possibility, if amount of information stored is a dominant factor in knowledge confidence, the habit of deleting files might be associated with reduced confidence. Conversely, if organization of information is the dominant factor, then deleting files should be associated with greater confidence, because deleting files culls the unneeded or irrelevant and neatens the repository. 


\section{Study 1}

\section{Method}

Participants. Participants for this study were 75 individuals recruited from the on-line research site Amazon Mechanical Turk (Buhrmester, Kwang, \& Gosling, 2011) and compensated $\$ 0.75$. Seven participants failed an attention check item embedded in one of the questionnaires. Therefore, the final sample contained 68 individuals (41 men, 27 women). Ages ranged from 19 to 63 years old $(M=33.07, S D=10.79)$, and all participants lived in the United States. Participants were also daily and experienced computer users, with estimated times ranging from 2 to 16 hours per day $(M=8.10, S D=3.19)$ and number of years of experience ranging from 3 to $30(M=18.51, S D=5.66)$.

Materials and procedure. Participants were first asked to judge confidence in knowledge by responding to the question "Considering everything you should know about your job (or courses), how knowledgeable do you feel?" Responses were recorded by dragging a slider across a scale that ranged from 0 to 100.

After responding to this question, participants were asked to think about the electronic repository where they had the most information stored (e.g., desktop, hard drive, Dropbox) and to answer several questions related to the way they use this area. The questions were designed to provide data regarding depth of organization, confidence in ability to quickly find files (CQF), degree of organization, and search method. Depth of organization assessed the depth to which participants used a vertical hierarchy of sub-folders to organize and retrieve files. $C Q F$ assessed the efficiency with which participants believed they could find their files. Degree of organization assessed the extent to which participants believed their files were well-organized. Search method assessed whether participants retrieved their files by manually clicking through folders, by using 
an automated search feature, or with some other search method. A complete list of questions and response scales are reported in the Appendix.

After answering the organization questions, participants completed the Cognitive SelfEsteem Scale (CSE; Ward, 2013). This 14-item scale measures participants' beliefs about their cognitive abilities and therefore is more general than the content-specific rating of knowledge confidence. The CSE scale contains three sub-components that assess confidence in the ability to think (e.g., "I am good at thinking"), to remember (e.g., "I have a better memory than most people"), and to locate information (e.g., "When I don't know the answer to a question right away, I know where to find it”). Responses were coded on a 7-point Likert scale ( 1 = Strongly Disagree to $7=$ Strongly Agree), such that higher ratings would indicate higher levels of CSE. The CSE scale demonstrated good reliability $(\alpha=.93) .{ }^{1}$ Last, we asked questions about age, gender, education, and computer experience (number of years of computer use, hours of use per day).

\section{Results and Discussion}

Prediction 1: Primary organization variables as predictors of knowledge confidence and CSE. Table 1 presents means, standard deviations, and bivariate correlations for ratings related to participants' organizational tendencies. In support of our main prediction, degree of organization of the participants' primary electronic repository was significantly and positively correlated with both knowledge confidence and CSE. Participants who judged their files as accurately located within the system they had created reported higher confidence in their knowledge and higher CSE. CQF was also positively correlated with knowledge confidence and CSE, a finding that supports the involvement of an availability heuristic; participants who were more confident in their ability to quickly find files reported greater confidence in their 
knowledge and higher CSE. Depth of organization did not significantly correlate with knowledge confidence or CSE, each $p>.75$.

Next, we performed a series of regression analyses to determine whether the relation between the measures of organization and the cognitive measures would remain significant when we entered the organization variables simultaneously into a regression equation. As shown in Table 1, although the overall model was significant, $F(3,64)=3.07, p=.034, R^{2}=.13$; none of the organization variables significantly contributed to the model independently from the others, each $p>.17 .^{2}$ A similar analysis conducted for CSE revealed that the overall model was also significant, $F(3,64)=5.06, p=.003, R^{2}=.19$; degree of organization was the only significant predictor, independent from the other variables in the model, $p=.041$. Depth of organization and CQF did not significantly contribute to the multiple-regression model, each $p>.21$. In short, the two main outcome variables, knowledge confidence and CSE, were somewhat differentially related to organizational variables. In that regard, it is important to note that the two outcome variables were only moderately correlated, $r(66)=.37, p=.002$.

Prediction 2: Search method as a moderator. To test our prediction that degree of organization is correlated with knowledge confidence, but only when the search method requires an organized repository, we conducted two moderation analyses using hierarchical regression in which we entered degree of organization and search method $(0=$ Click through folders, $1=$ Use a search feature) in the first step and their interaction in the second step, with knowledge confidence and CSE as the dependent variables in separate analyses. ${ }^{3}$

Moderation analysis for knowledge confidence. The model in the first step was significant, $F(2,64)=4.47, p=.015, R^{2}=.12$, and degree of organization was the only significant predictor of knowledge confidence $(\beta=.36, t=2.96, p=.004)$. As predicted, 
however, the model was significantly improved in the second step when the Degree of Organization $\times$ Search Method interaction term was included, $\Delta F(1,63)=4.68, p=.034, \Delta R^{2}=$ .06; The interaction term was a significant predictor of knowledge confidence after allowing for the correlation with knowledge confidence. As shown in Figure 1, individuals who manually click through folders $(n=44)$ reported significantly higher knowledge confidence if they had also reported a higher degree of organization $(\beta=.50, t=3.75, p=.001)$, whereas in the case of individuals who reported use of an automated search feature $(n=23)$, the relation between knowledge confidence and degree of organization was nonsignificant $(\beta=.16, t=.72, p=.481)$.

Moderation analysis for CSE. A similar approach was taken with CSE as the outcome variable. The model in the first step was significant, $F(2,64)=7.40, p=.001, R^{2}=.19$. Only degree of organization was a significant predictor of $\operatorname{CSE}(\beta=.44, t=3.82, p<.001)$. In the second step, the interaction term did not account for a significant increase in variance in CSE, $\Delta$ $F(1,63)=1.83, p=.181, \Delta R^{2}=.023$. Thus, even though the pattern was similar to the one found for knowledge confidence, search method did not significantly moderate the relationship between degree of organization and CSE.

Exploratory analyses. In addition to the primary organization questions, participants were asked questions related to the way they store information on their computer desktops. The three variables of interest included number of desktop items, desktop arrangement, and desktop cleanliness (see the Appendix). None of these variables significantly predicted knowledge confidence, each $p>.37$. However, desktop arrangement $(0=$ Dispersed to $100=$ Clustered $)$ was significantly and positively related to CSE, $r(58)=.36, p=.005$, indicating that individuals who arranged their files in organized clusters (vs. leaving files dispersed) tend to report higher CSE. Furthermore, the overall model was significant, $F(3,56)=2.87, p=.044, R^{2}=.13$; desktop 
arrangement was a significant predictor, independent from the number of desktop items and desktop cleanliness.

To account for the possibility that older participants might be more experienced in their work, and therefore, more confident in their knowledge; we calculated bivariate correlations between age and each outcome variable. Results revealed a significant correlation between age and knowledge confidence such that older participants tend to be more confident in their knowledge related to their work, $r(68)=.29, p=.016$. The relationship between age and CSE was not significant, $r(68)=.20, p=.097$.

Given that age was a significant predictor of knowledge confidence, we next examined whether age interacted with degree of organization and search method. To test this possibility, we conducted a multiple regression in which we entered degree of organization, age (split at the median), search method, and the three-way interaction term simultaneously into the regression model. Overall, the model was significant, $F(4,64)=3.90, \mathrm{p}=.007, R^{2}=.21$; only age $(\beta=$ $.25, p=.038)$ and degree of organization $(\beta=.25, p=.028)$ emerged as significant predictors of knowledge confidence.

Summary. The main results of Study 1 support our predictions and suggest that the degree to which individuals organize computer files and can find them quickly significantly predicts their knowledge confidence and cognitive self-esteem. Furthermore, moderation analyses reveal that the relation between knowledge confidence and degree of organization was moderated by search method, such that the relationship was significant when participants navigated by manually clicking through folders to find their files, but not when participants used automated search features to find their files. Desktop organization also predicted CSE, although 
it did not predict knowledge confidence. Finally, age significantly predicted knowledge confidence, but did not interact with organization and search method.

In Study 2 we attempted to replicate the association between organization of external repositories and knowledge confidence, and we addressed new exploratory questions related to keeping and deleting files. Responses to these questions potentially reveal other aspects of the use of an organization heuristic to judge knowledge. Namely, if a positive relation between deleting files and knowledge confidence or CSE appears, it provides indirect evidence for an organization heuristic in the sense that individuals who delete useless items more often have better-organized repositories. If a negative relation appears, however, it might suggest that knowledge confidence or CSE instead reflects the overall amount of information stored.

\section{Study 2}

\section{Method}

Participants. Participants for this study were 79 students recruited from psychology classes. Participants received credit toward the partial fulfillment of a course requirement. Thirteen participants failed the attention check. Therefore, the final sample contained 66 individuals (18 men, 48 women). Ages ranged from 18 to $22(M=19.28, S D=1.23)$. The majority of the participants reported a European Ancestry (54.5\%), followed by Hispanic/Latino/Spanish (27.3\%), Asian (15.2\%), African (9.1\%), and Other (7.2\%). Participants reported daily computer use $(M=6.34, S D=3.01)$ and about 10 years of computer experience, on average $(M=9.86, S D=3.25)$.

Materials and procedure. As in Study 1, participants were first asked to judge their confidence in knowledge related to their school courses followed by the questions from Study 1 that concerned organizational tendencies. Then we asked them why they choose to keep certain 
files, how often they delete files, and what they do with files once they complete an assignment or course (items reported in the Appendix.) Following these questions, participants completed the CSE $(\alpha=.87)$ and answered the demographic questions.

\section{Results and Discussion}

Prediction 1: Primary organization variables as predictors of knowledge confidence

and CSE. Table 2 presents means, standard deviations, and bivariate correlations for ratings related to participants' organizational tendencies. In support of our predictions and replicating the results of Study 1, degree of organization was significantly and positively related to knowledge confidence, indicating that participants with better organized files were also more confident in knowledge related to their courses, $p=.011$. In the corresponding regression analysis, the overall model was significant, $F(2,63)=5.10, p=.009, R^{2}=.14$; degree of organization was the only significant predictor $(\beta=.51, p=.003)$. When the same analyses were performed with CSE as the outcome variable, the zero-order correlations and the model were nonsignificant, each $p>.11$.

Prediction 2: Search method as a moderator. In Study 1, search method moderated the association between degree of organization and knowledge confidence. To assess replication, we again conducted hierarchical regressions in which we entered degree of organization and search method ( $n=47$ click through folders; $n=18$ use a search feature) in the first step and a term representing their interaction in the second step. Unlike Study 1, the interaction term failed to account for a significant increase in explained variance in either knowledge confidence or CSE, $p>.32$. Again, knowledge confidence and CSE were not highly correlated, $r(64)=.28, p=.023$.

Exploratory analyses. In addition to the primary organization questions, participants answered questions related to their tendency to keep or delete files on their computers. None of 
the deletion-related variables significantly predicted knowledge confidence, $p>.14$. However, bivariate correlations indicate that participants' tendency to delete course-related files and completed assignments was associated with higher CSE, $r(64)=-.26, p=.036$, and $r(64)=-.32$, $p=.008$, respectively. Choosing to keep files in anticipation of future use was positively correlated with CSE, $r(62)=.34, p=.007$, whereas choosing to keep files because they were personally meaningful was not, $r(61)=.02, p=.861$. In addition, participants who reported being more organized also reported deleting files that are not important to them more often, $r(64)$ $=.32, p=.008$. More generally, if judgments of knowledge or CSE were primarily a reflection of the amount of information stored, we should expect that individuals with greater confidence or self-esteem would be more likely to keep files after completing an assignment. However, these results showed the opposite relation. Students with higher cognitive self-esteem more often delete files that are no longer important.

Internal meta-analysis. In a final set of analyses, we performed Fisher $r$-to- $z$ transformations to determine whether the correlations observed in Studies 1 and 2 were consistent with one another. In support of our conclusions, both the correlations between organization and knowledge confidence, $z=.06, p=.95$ and organization and cognitive selfesteem, $z=1.38, p=.17$ were not significantly different, indicating that the results were consistent across the two studies.

Given that the results were consistent, we next conducted an internal meta-analysis to determine the overall robustness of the observed associations, using Comprehensive MetaAnalysis (Version 2.2.064; Borenstein, Hedges, Higgins, \& Rothstein, 2005). The results of this analysis revealed a moderate correlation between organization and knowledge confidence, $r=$ $.31, p<.001,95 \% \mathrm{CI}=.15$ to .46 , as well as a moderate correlation between organization and 
cognitive self-esteem, $r=.30, p<.001,95 \% \mathrm{CI}=.41$ to .45 . These results provide clear evidence for the associations between organization and our primary outcome variables.

\section{General Discussion}

The results of the current investigation are consistent with the notion that the organization of external memory is associated with judgments of personal knowledge, and extend earlier research on physical repositories (Hertel, 1988) to digital domains. Relying on ease of access produces judgment bias (Tversky \& Kahneman, 1973), and relying on sense of organization might do the same. We hypothesized that organizational tendencies would be associated with higher confidence in knowledge related to one's main intellectual enterprise and higher cognitive selfesteem. In support of this hypothesis, both studies revealed that individuals who maintained betterorganized electronic repositories were also more confident in their knowledge and reported higher CSE.

We also predicted that organization cues would be used to the extent that the method used to retrieve electronic information actually requires an organized repository. As predicted, the relation in Study 1 between perceived organization and knowledge confidence was stronger for individuals who navigate through their files by manually clicking through folders than for those who use automated search functions. In the student sample for Study 2, however, search method did not play a moderating role. Although age did not interact with search method in Study 1, we can speculate that cohort differences might be related to the moderation differences across the studies, the average participant in Study $1(M=33.07)$ was much older than the average age of participants in Study $2(M=19.28)$. For example, one of the co-authors uses a detailed system for labeling files in order to quickly retrieve files via an automated search feature. In this sense, more efficient searchers may not demand an organized repository. It is also 
possible that search method did not play a moderating role in our student sample because this sample was comprised of first-year undergraduate student, who are unlikely to use a sophisticated approach. Moreover, students may not have had the opportunity to develop organization schemes to the extent that search method becomes a relevant moderator of perceived knowledge, despite perceived organization significantly correlating with knowledge confidence.

We also explored additional variables related to file organization. In Study 1, individuals who arranged their desktop files in organized clusters (vs. leaving files dispersed) tend to report higher CSE; files that are organized spatially in clusters are more likely to be easily located. In Study 2, individuals who reported deleting more files related to courses and assignments once completed also reported higher CSE. Again, this finding supports the importance of organization, because deleting irrelevant files facilitates retrieval of the relevant.

Self-perceptions of knowledge can be captured in a variety of ways, all of which represent slightly difference constructs. In this study, we examined two main outcome variables: knowledge confidence ("Considering everything you should know about your job (or courses), how knowledgeable do you feel?" and the more general measure of cognitive self-esteem (CSE; Ward, 2013); the former measure refers to a specific domain of knowledge, whereas CSE is a collection of more global judgments about self. This difference might help us understand their differential relation to organizational variables. For example, we found that search method moderated the relation between degree of organization and knowledge confidence but not CSE because we don't search for global attributes. Instead, CSE was related to the tendency in Study 2 for students to either keep or delete files; this tendency seems to be a more global judgment of their external-memory habits. 
In a speculative vein we suggest that personal computers might lead to more source confusion than do other modern external repositories, such as the internet, due to a mere ownership effect (Kim \& Johnson, 2013). Because individuals include self-relevant others or objects in one's sense of self (Kim \& Johnson, 2013), when an external repository becomes a 'personal' external repository, the likelihood of conflating internal and external knowledge during metacognitive judgments might be higher. Thus, while previous research has shown that the relative familiarity of common access points to the internet (e.g., Google) lead to more frequent misattributions of information than do uncommon access points (e.g., Lycos; Ward, 2013), through a similar logic, it could be argued that personally-relevant access points could lead to more frequent misattributions of information than public access points. This distinction is worth pursuing experimentally.

An important limitation of the current findings concerns our difficulty in evaluating the importance of the organization of information independently from the amount of information stored. The exploratory analyses in Study 2 found that individuals with better-organized repositories tend to delete their assignments, and they also reported higher cognitive self-esteem. Nevertheless, it would be worthwhile to examine the predictive value of organization over and above the amount of information stored, even though valid measures of the latter would be very difficult to develop.

An additional limitation of the current investigation was its correlational nature. We cannot say whether degree of organization facilitates knowledge confidence, confidence in knowledge increases one's tendency to organize, or some third variable, like expertise, affects both confidence and organization. As is true of other complex systems, multiple directions of cause are likely. To assess the causal contribution of organization to confidence, future research 
might include manipulations of external organization. Such efforts, however, will likely suffer the loss of ecological validity critical to the examination of cognitive influences of digital storage. Therefore, other approaches — such as the moderation effect in Study 1 - might provide indirect support for organization as a contributory cause of confidence.

In conclusion, our evidence replicates earlier findings concerning non-digital external repositories (Hertel, 1993) and knowledge appropriated from the internet (Ward, 2013). More generally, the blurring of distinctions between organic and inorganic "stores" of knowledge as individual attempt to make knowledge judgments extends the reality-monitoring framework (Johnson \& Raye, 1981) to issues related to accessibility. As new technology narrows the bases for making distinctions (the phone becomes a body part?), we can expect it to become even more difficult to know what we know. 


\section{References}

Borenstein, M., Hedges, L.V., Higgins, J.P.T., \& Rothstein, H.R. (2005). Comprehensive metaanalysis version 2. Englewood, NJ: Biostat.

Bower, G. H. (1970). Organizational factors in memory. Cognitive Psychology, 1(1), 18-46.

Buhrmester, M. D., Kwang, T., \& Gosling, S. D. (2011). Amazon's Mechanical Turk: A new source of inexpensive, yet high-quality, data? Perspectives on Psychological Science, 3, 3-5.

Duckworth, A. L., Peterson, C., Matthews, M. D., \& Kelly, D. R. (2007). Grit: perseverance and passion for long-term goals. Journal of Personality and Social Psychology, 92(6), 1087.

Fisher, M., Goddu, M. K., \& Keil, F. C. (2015). Searching for explanations: How the Internet inflates estimates of internal knowledge. Journal of Experimental Psychology: General, 144(3), 674-687. doi:10.1037/xge0000070

Gosling, S. D., Rentfrow, P. J., \& Swann, W. B. (2003). A very brief measure of the Big-Five personality domains. Journal of Research in Personality, 37(6), 504-528.

Hertel, P.T. (1988). Monitoring external memory. In M. M. Gruneberg, P. E. Morris \& R. N. Sykes (Eds), Practical aspects of memory: Current research and issues (pp. 221-226). New York: Wiley.

Hertel, P. T. (1993). Implications of external memory for investigations of mind. Applied Cognitive Psychology, 7(7), 665-674.

Johnson, M. K., \& Raye, C. L. (1981). Reality monitoring. Psychological Review, 88(1), 67.

Kelley, C. M., \& Lindsay, D. S. (1993). Remembering mistaken for knowing: Ease of retrieval as a basis for confidence in answers to general knowledge questions. Journal of Memory and Language, 32(1), 1-24. 
Kim, K., \& Johnson, M. K. (2013). Extended self: Medial prefrontal activity during transient association of self and objects. Social Cognitive and Affective Neuroscience, 7(2), 199207. doi:10.1093/scan/nst082

Mitchell, K.J., \& Johnson, M.K. (2000). Source monitoring: Attributing mental experiences. In E. Tulving \& F.I.M. Craik (Eds.), Oxford Handbook of Memory (pp. 179-195). New York: Oxford University Press.

Tversky, A., \& Kahneman, D. (1973). Availability: A heuristic for judging frequency and probability. Cognitive psychology, 5(2), 207-232.

Ward, A. F. (2013). Supernormal: How the Internet is changing our memories and our minds. Psychological Inquiry, 24(4), 341-348.

Winkielman, P., Schwarz, N., \& Belli, R. F. (1998). The role of ease of retrieval and attribution in memory judgments: Judging your memory as worse despite recalling more events. Psychological Science, 9(2), 124-126. 


\section{Footnotes}

${ }^{1}$ The 10-item Personality Inventory (Gosling, Rentfrow, \& Swann, 2003) and the Grit Scale (Duckworth, Peterson, Matthews, \& Kelly, 2007) were also used in Study 1, but were not relevant to the predictions described in this report.

${ }^{2}$ Although depth of organization was not significantly correlated with the other two organization predictors, $p>.48, \mathrm{CQF}$ and degree of organization were highly correlated, $r(66)=$ $.63, p<.001$.

${ }^{3}$ Participants responded based on how they typically search for files, however people may use both methods to different extents. One participant used an "indexing program" to search for files and was excluded from the data analyses. Search method and degree of organization were not highly correlated, $r(67)=-.22, p=.080$, degree of organization for those reporting clicking through files $(M=81.4)$ and for those reporting the use of a search feature $(M=72.6)$. 
Appendix

Survey Questions

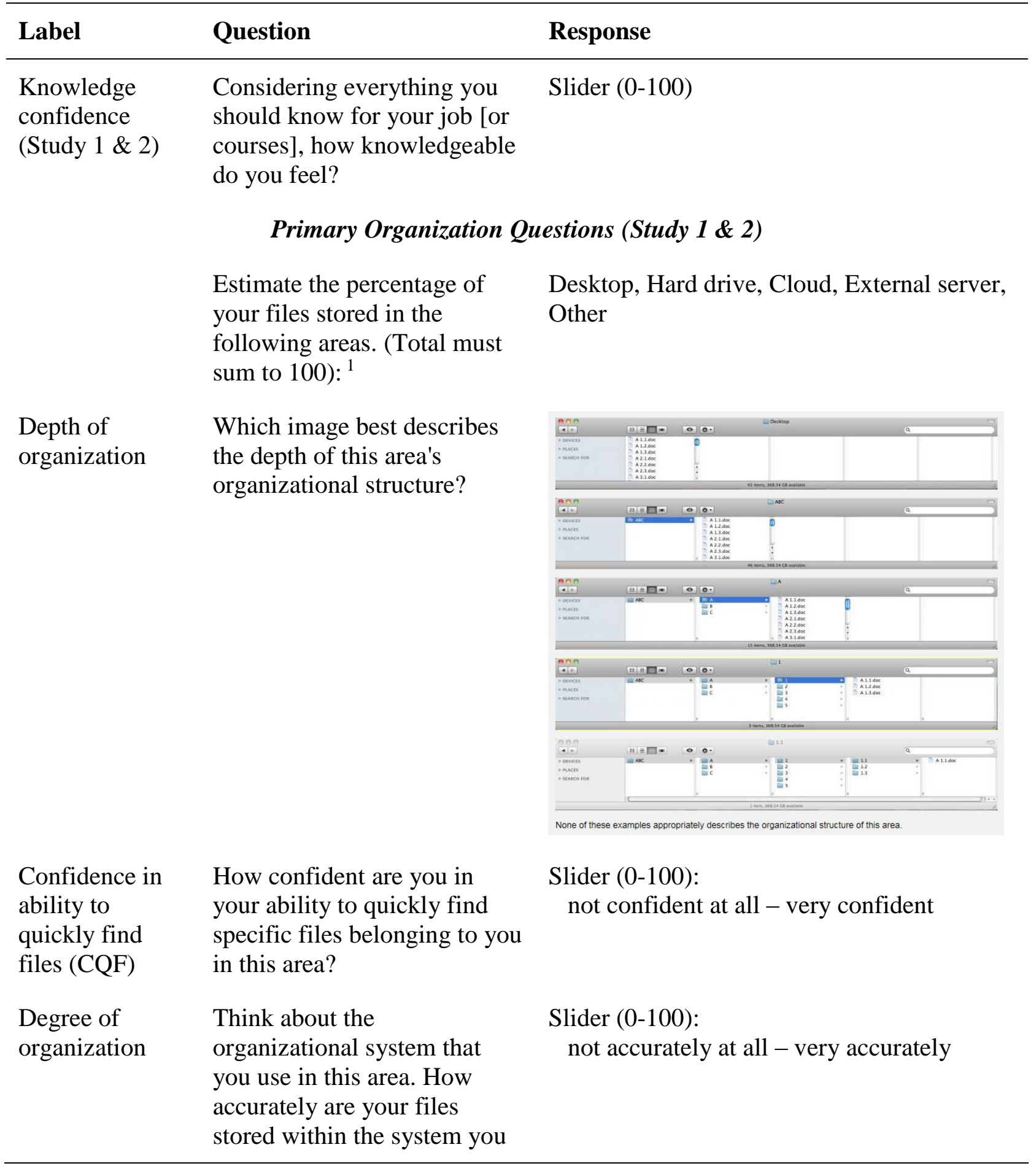

\footnotetext{
${ }^{1}$ Participants were told to refer to the area that they have indicated they have stored the most files on for the following questions on the page.
} 


\begin{tabular}{|c|c|c|}
\hline & $\begin{array}{l}\text { described (i.e., Is everything } \\
\text { where it's supposed to be)? }\end{array}$ & \\
\hline Search method & $\begin{array}{l}\text { How do you typically search } \\
\text { for specific files in this area? }\end{array}$ & $\begin{array}{l}\text { click through folders, use a search feature, } \\
\text { other }\end{array}$ \\
\hline \multicolumn{3}{|c|}{ Desktop (Study 1) } \\
\hline $\begin{array}{l}\text { Number of } \\
\text { desktop items }\end{array}$ & $\begin{array}{l}\text { How many items do you have } \\
\text { on your desktop? }\end{array}$ & (Space for text entry) \\
\hline \multirow[t]{2}{*}{$\begin{array}{l}\text { Desktop } \\
\text { arrangement }\end{array}$} & $\begin{array}{l}\text { How are related items on your } \\
\text { desktop arranged? }\end{array}$ & Slider (0-100): dispersed - clustered \\
\hline & $\begin{array}{l}\text { Briefly describe the way your } \\
\text { files are arranged on your } \\
\text { desktop. }\end{array}$ & (Space for text entry) \\
\hline \multirow[t]{6}{*}{$\begin{array}{l}\text { Desktop } \\
\text { cleanliness }\end{array}$} & $\begin{array}{l}\text { How often do you clean your } \\
\text { desktop? }\end{array}$ & Slider (0-100): never - every day \\
\hline & \multicolumn{2}{|c|}{ Keeping/Deleting Files (Study 2) } \\
\hline & $\begin{array}{l}\text { How often do you delete files } \\
\text { that are not important to you? }\end{array}$ & Slider (0-100): never - every day \\
\hline & $\begin{array}{l}\text { What do you typically do } \\
\text { with files related to an } \\
\text { assignment once the } \\
\text { assignment is completed? }\end{array}$ & Slider (0-100): delete all files - store all files \\
\hline & $\begin{array}{l}\text { What do you typically do } \\
\text { with files from a course once } \\
\text { the course is completed? }\end{array}$ & Slider (0-100): delete all files - store all files \\
\hline & $\begin{array}{l}\text { Why do you choose to keep } \\
\text { the files that you keep? } \\
\text {-I might want to use it again } \\
\text {-It is personally meaningful to } \\
\text { me }\end{array}$ & Slider $(0-100)$ \\
\hline
\end{tabular}


Table 1

Study 1: Descriptive Statistics, Correlations, and Multiple-Regression Statistics

\begin{tabular}{lrrrrr}
\hline & Mean & SD & $r$ & $\beta$ & $R^{2}$ \\
\hline Knowledge confidence & 78.07 & 18.55 & & & $.126^{*}$ \\
Degree of organization & 78.48 & 19.34 & $.32^{* *}$ & .19 & \\
Depth of organization & 3.50 & 1.40 & -.01 & -.03 & \\
CQF & 75.96 & 23.54 & $.32^{* *}$ & .21 & \\
CSE & 5.41 & .92 & & & $.192^{* *}$ \\
Degree of organization & & & $.41 * *$ & $.30 *$ & \\
Depth of organization & & & -.04 & -.07 & \\
CQF & & & $.36 * *$ & .18 & \\
\hline
\end{tabular}

Note. All ratings are based on a 0-100 slider scale except CSE (7-pt scale). CSE = Cognitive Self-Esteem. $\mathrm{CQF}=$ Confidence in the ability to quickly find files. $N=68,{ }^{*} p<.05, * * p<.01$. 
Table 2

Study 2: Descriptive Statistics, Correlations, and Multiple-Regression Statistics

\begin{tabular}{lrrrrr}
\hline Outcome/predictors & $M$ & $S D$ & $r$ & $\beta$ & $R^{2}$ \\
\hline Knowledge confidence & 71.89 & 15.77 & & & $.140^{* *}$ \\
Degree of organization & 77.62 & 23.37 & $.31^{*}$ & $.51^{* *}$ & \\
CQF & 82.42 & 18.94 & .07 & -.29 & \\
CSE & 5.29 & .73 & & & .044 \\
Degree of organization & & & .19 & .12 & \\
CQF & & & .19 & .11 & \\
\hline
\end{tabular}

Note. All ratings are based on a 0-100 slider scale except CSE (7-pt scale). CSE = Cognitive

Self-Esteem. $\mathrm{CQF}=$ Confidence in the ability to quickly find files. $N=66, * p<.05, * * p<.01$. 


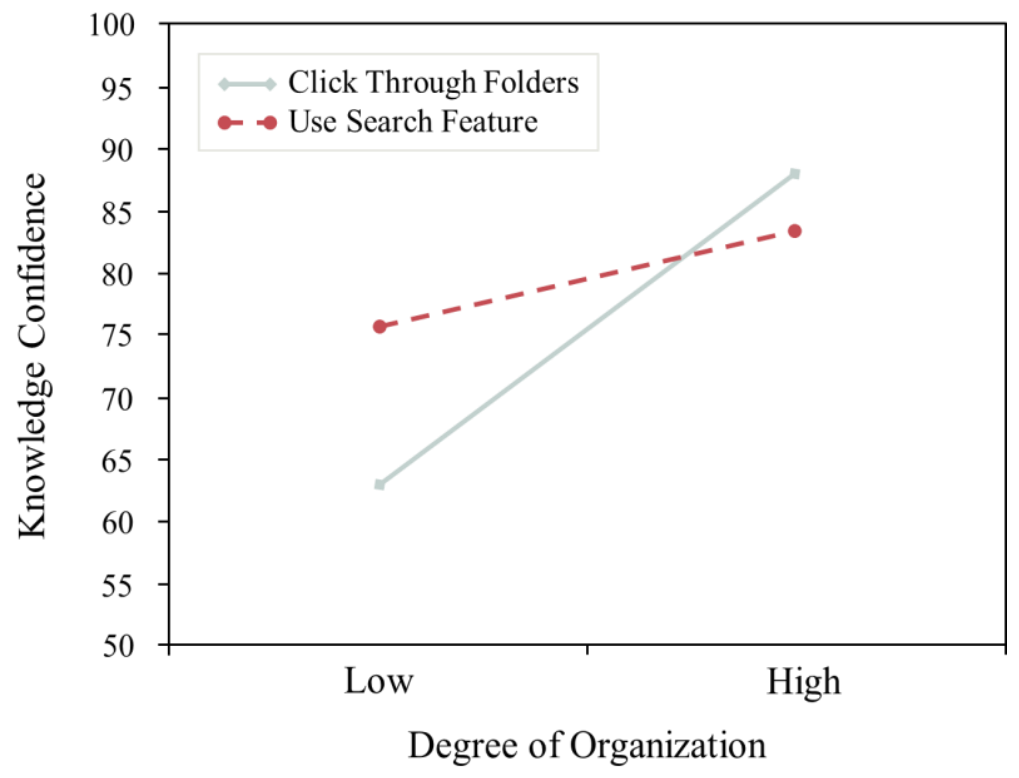

Figure 1. Search method moderates the association between degree of organization and knowledge confidence. 